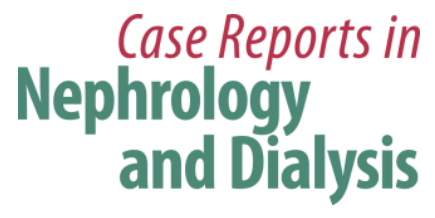

Case Rep Nephrol Dial 2018;8:182-191

DOI: 10.1159/000492529

Published online: August 31, 2018

(c) 2018 The Author(s)

Published by S. Karger AG, Basel

www.karger.com/cnd

This article is licensed under the Creative Commons Attribution-NonCommercial 4.0 International License (CC BY-NC) (http://www.karger.com/Services/OpenAccessLicense). Usage and distribution for commercial purposes requires written permission.

\title{
Fibrillary Glomerulonephritis with Crescentic and Necrotizing Glomerulonephritis and Concurrent Thrombotic Microangiopathy
}

\author{
Calvin Tsui $^{\mathrm{a}}$ Pouneh Dokouhaki $^{\mathrm{b}} \quad$ Bhanu Prasad ${ }^{\mathrm{c}}$ \\ aCollege of Medicine, University of Saskatchewan, Regina, SK, Canada; ${ }^{b}$ Department of \\ Pathology and Laboratory Medicine, College of Medicine, University of Saskatchewan, \\ Saskatoon, SK, Canada; 'Regina General Hospital, Section of Nephrology, Department of \\ Medicine, Regina, SK, Canada
}

\section{Keywords}

Fibrillary glomerulonephritis · Thrombotic microangiopathy · Crescentic changes

\begin{abstract}
We present a 77-year-old Caucasian woman who presented with nephrotic-range proteinuria, microhematuria, renal impairment, and extremely elevated blood pressure. She had a long history of well-controlled type 2 diabetes. Renal biopsy revealed fibrillary deposits in the mesangium and glomerular basement membrane consistent with fibrillary glomerulopathy (FGN), with crescentic changes and thrombotic microangiopathy (TMA). We could not identify any radiological, clinical, or laboratory evidence of autoimmune disorders, lymphoproliferative disorders, and malignancy. It was decided not to offer her any immunosuppressive therapy, as she was frail with substantial renal damage on the biopsy. Five months after presentation, she gradually progressed to requiring renal replacement therapy and is currently on maintenance hemodialysis. Crescentic changes in FGN, though rare, have been previously described, but the concurrent presence of TMA has never been previously reported.
\end{abstract}




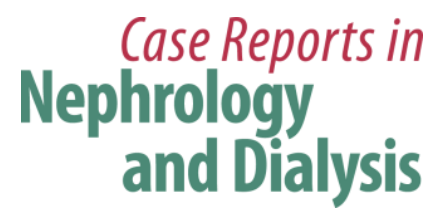

Case Rep Nephrol Dial 2018;8:182-19

DOI: $10.1159 / 000492529$

(C) 2018 The Author(s). Published by S. Karger AG, Base www.karger.com/cnd

Tsui et al.: FGN with Crescentic and Necrotizing Glomerulonephritis and Concurrent TMA

\section{Introduction}

Fibrillary glomerulonephritis (FGN) is a rare primary glomerular disorder that occurs in approximately $0.6-1.0 \%$ of primary glomerular disorders identified on a native kidney biopsy [1]. FGN presents akin to most glomerular diseases with nephrotic-range proteinuria, renal impairment, microscopic hematuria, and hypertension. The diagnosis of FGN is made on a kidney biopsy. In particular, changes on electron microscopy showing randomly aligned fibrils of specific diameter (10.0-18.0 nm) along with direct immunofluorescence staining with or without complement are confirmatory of diagnosis [1].

FGN on light microscopy typically presents as normocellular glomeruli with deposition of typical PAS-positive and silver-negative material in the mesangium and along glomerular basement membranes. These features mimic diabetic nephropathy by light microscopy, rendering the diagnosis of FGN in a diabetic patient challenging [2]. Our patient presented similarly with changes suggestive of nodular mesangial expansion, but immunofluorescence showed smudgy IgG and complement (C3 and C1q) staining in the glomeruli, and electron microscopy confirmed the presence of typical fibrils. There were also crescentic changes on the biopsy and more surprisingly, there were vascular changes consistent with thrombotic microangiopathy (TMA). This is the first case report which shows concurrent TMA and crescentic FGN.

\section{Case Report}

This 79-year-old female was referred by her family physician to the nephrology service when it was noticed that the blood pressure was $220 / 120 \mathrm{~mm} \mathrm{Hg}$, incidental creatinine was $250 \mu \mathrm{mol} / \mathrm{L}(140 \mu \mathrm{mol} / \mathrm{L} 6$ months ago), and urine analysis was positive for blood and protein. On microscopy, there were $>40$ red cells/hpf and upon quantification, there was $6.6 \mathrm{~g} /$ day of proteinuria, with edema extending up to the shins. Her past medical history was significant for well-managed type 2 diabetes and dyslipidemia. She claimed worsening control of blood pressure 6 months prior to her presentation but failed to seek medical attention. She had no known history of malignancy, autoimmune disease such as scleroderma, or systemic lupus erythematosus. She denied constitutional symptoms, hemoptysis, bloody nasal/sinus discharge, rash, or joint effusions but complained of 30-pound weight loss over 12 months. On clinical examination, the average of six blood pressure readings on the left brachial artery on BP Tru was 191/112 mm Hg and on the right side was 194/140 mm Hg. Cardiac exam was unremarkable; chest was clear to auscultation and abdomen was soft and non-tender. There was peripheral edema (left greater than right) extending up to mid shins. She was on metformin $500 \mathrm{mg}$ three times per day for control of diabetes, amlodipine $10 \mathrm{mg}$ once a day for hypertension, and rabeprazole $20 \mathrm{mg}$ once a day for control of symptoms of acid reflux. She was initiated on telmisartan $80 \mathrm{mg}$ a day, bisoprolol $5 \mathrm{mg}$ a day, and clonidine $0.1 \mathrm{mg} 3$ times a day for blood pressure control, and the kidney biopsy was delayed till the blood pressure control improved to decrease the risk of a post-biopsy bleed.

Due to her history of 30-pound weight loss, she underwent an upper gastrointestinal endoscopy and colonoscopy, which were negative for malignancy. She also underwent a CT of the chest and abdomen, which did not show any evidence of malignancy, but the kidneys were 


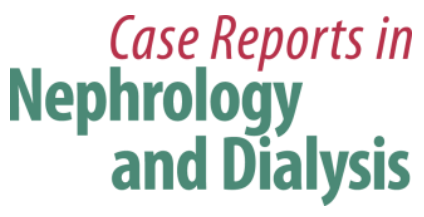

Case Rep Nephrol Dial 2018;8:182-191

large with multiple cysts within both kidneys. The left kidney measured $14.6 \mathrm{~cm}$ and the right measured $10.2 \mathrm{~cm}$. An incidental note was made of aneurysmal dilation of the abdominal aorta measuring $3.7 \mathrm{~cm}$. There was an area of collapse/consolidation involving the left anterior and medial basal segments. The presence of multiple renal cysts was pursued further for autosomal dominant polycystic kidney disease. However, there was no history of polycystic disease in the family and her children had undergone abdominal ultrasound investigations for other clinical indications and were not found to have cysts in the kidney.

Due to the presence of proteinuria and microhematuria, acute kidney injury on a background of chronic renal impairment, she underwent a kidney biopsy, which showed features suggestive of TMA likely due to her recent uncontrolled hypertension (Fig. 1a, b). The glomeruli showed no prominent hypercellularity but exhibited collapsed capillaries and prominence of epithelial cells (Fig. 1c, d). The conspicuous finding was the focal segmental necrotizing and crescentic glomerulonephritis with expansion of mesangium with PAS-positive, partially silver-negative material (Fig. 2). Chronic glomerular changes consistent with diabetic nephropathy class IIb were present on the background and they were accompanied by diffuse mild acute tubular injury and multifocal acute interstitial nephritis. Moderate arteriosclerosis and moderate to severe arterial hyalinosis were indicative of a long-lasting hypertensive and diabetic nephropathy. Congo-red stain was negative under polarized light. Immunofluorescence microscopy revealed segmental smudgy deposition of IgG, C3, and to a lesser degree C1q (Fig. $3 a-c)$. Electron microscopy was diagnostic for fibrillary deposits in mesangium and along the glomerular basement membranes. The fibrils were arranged haphazardly and measured slightly thicker than amyloid fibrils with the thickness range of 10.4-14.8 nm (average: 12.4 $\mathrm{nm}$ ). No substructural pattern was identified (Fig. 3d-f). The representative images of higher magnification of electron microscopy of the fibrillary deposits are included (Fig. 4). Immunohistochemistry staining for DNAJB9 - a newly discovered marker specific for FGN - revealed strong positivity in the mesangial and basement membrane deposits confirming the diagnosis of FGN.

She underwent investigations including anti-nuclear antibody (ANA), anti-nuclear cytoplasmic antibody (ANCA), anti-glomerular basement membrane antibody (anti-GBM), C3-C4, serum protein electrophoresis, cryoglobulins, hepatitis B, hepatitis C, and HIV (Table 1). Her peripheral blood was investigated further by flow cytometry, which showed $34 \%$ B cells, $45.25 \% \mathrm{~T}$ cells, and $21.2 \% \mathrm{NK}$ cells. Serum $\kappa$ light chains were $107.0 \mathrm{mg} / \mathrm{L}(3.3-19.4), \lambda$ light chains were $66.6 \mathrm{mg} / \mathrm{L}$ (5.7-26.3), and $\kappa-\lambda$ ratio was 1.61. A repeat review of the CT of the chest, abdomen, and pelvis was undertaken with the radiologist and we were reliably informed that there was no apparent lymphoma or malignancy. While there was no evidence of malignancy, there was presence of severe multilevel degenerative back disease and an incidental left ureteropelvic junction obstruction, which was successfully stented. We also aspirated $150 \mathrm{~mL}$ of serous fluid from the 2 largest cysts in the left kidney. The insertion of the stent along with drainage of the cysts led to an improvement in her back pain and resulted in better quality of life.

Her renal biopsy findings were consistent with FGN, which is an uncommon cause of glomerular disease (Table 2). In the patient's case, it occurred on a background of extensive damage from diabetes, hypertension, and atherosclerosis.

Unfortunately, there are no definite therapeutic options available for FGN. The therapeutic response of standard immunosuppressive agents (prednisone, cyclophosphamide and 


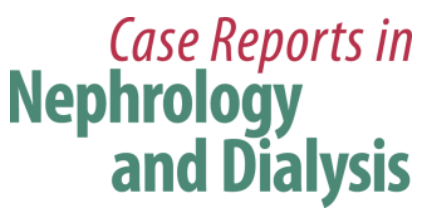

Case Rep Nephrol Dial 2018;8:182-191

DOI: 10.1159/000492529

(c) 2018 The Author(s). Published by S. Karger AG, Base www.karger.com/cnd

Tsui et al.: FGN with Crescentic and Necrotizing Glomerulonephritis and Concurrent TMA

cyclosporine, rituximab) has been variable and most people progress to end-stage renal disease. Our patient had clinical evidence of severe renal injury as suggested by a creatinine of $265 \mu \mathrm{mol} / \mathrm{L}$ (weight $55 \mathrm{~kg}$ ). Furthermore, the biopsy report suggested extensive glomerular sclerosis, tubular atrophy, and interstitial fibrosis. We pondered about exposing this frail elderly lady to a cocktail of immunosuppressive agents, and it was collaboratively decided that the risk would almost certainly exceed any potential benefit. She is also not a candidate for renal transplant given her advanced age and comorbid conditions. She was counselled on the possibility of requiring renal replacement therapy and 5 months post diagnosis ended up on hemodialysis. She is currently in a care home.

\section{Discussion}

FGN is a disease characterized by Congo-red-negative fibrillary deposits in the glomeruli. The incidence of FGN in native renal biopsies is only $0.8-1.5 \%[2,3]$. Most patients with idiopathic FGN present with nephrotic-range proteinuria, microhematuria, and decreased glomerular filtration rate. In the absence of effective pharmacotherapeutic options, the average time to progression to end-stage renal disease is $2-4$ years [3].

The pathological hallmark is fibrillary (straight, non-branching, and randomly oriented) deposits in the mesangium and glomerular basement membrane. A third of the patients have associated systemic illnesses such as hematologic malignancies, monoclonal gammopathies, chronic infections, and autoimmune disorders [4]. In our patient, we could not identify a malignancy following an upper gastrointestinal endoscopy, colonoscopy, and CT of the chest, abdomen, and pelvis. Serology for autoimmune diseases was negative and there was no evidence of monoclonal gammopathies as suggested by a negative serum protein electrophoresis and an unremarkable serum free light chain assay. The patient further underwent interrogation of peripheral blood for flow cytometry, which did not contribute further to the diagnosis.

The biopsy showed presence of background diabetic changes and there was extensive arteriosclerosis in keeping with her long history of smoking and hypertension. There was evidence of superimposed changes of FGN with crescentic changes and TMA, which we felt was consistent with the extremely elevated blood pressure of 200 systolic. TMA is a pattern of endothelial damage that can be found in association with diverse clinical conditions including malignant hypertension. TMA likely occurs when autoregulation fails to counteract the hypertension-induced shear stress [5]. The role of alternate pathway (AP) in TMA has been increasingly recognized over the last decade. AP is a continuously active immune surveillance and effector system operating in circulation and on the cell surface, which is tightly regulated to prevent damage to the self. AP dysregulation can be due to mutations in genes that either regulate or activate AP and/or autoantibodies that inhibit complement-regulatory proteins [5]. Clinically, it is important to distinguish between hypertension-induced TMA, atypical hemolytic uremic syndrome (caused by dysregulation of the AP pathway), and thrombotic thrombocytopenia [5].

While we predominantly focused on her underlying FGN, we felt that her TMA was renal limited (no drop in hemoglobin, platelets, or presence of schistocytes). Her biopsy had changes consistent with chronic hypertension and we presumed that TMA was due to hypertension-induced shear stress to the arteriole vasculature. We did not investigate with 

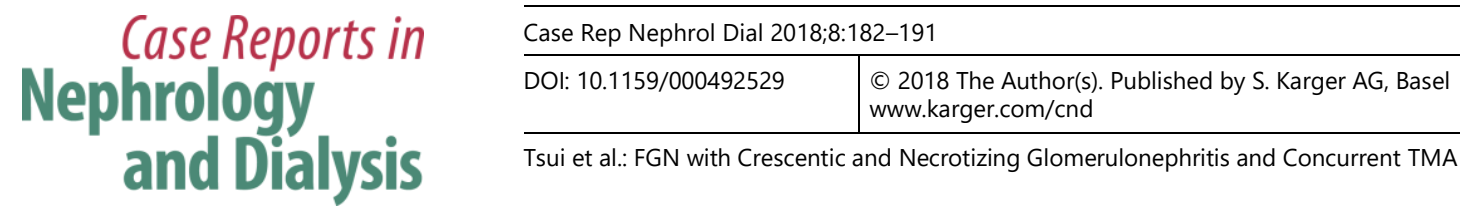

ADAMTS-13 levels or send labs for evaluation of the alternate complement pathway. We now recognize that renal TMA with severe hypertension and subtle signs of microangiopathic hemolytic anemia (MHA) can be mislabeled as malignant hypertension and conceal atypical hemolytic uremic syndrome, potentially jeopardizing renal recovery by withholding complement targeting drugs such as eculizimab.

Consistent with previous studies [6], the patient continued to have progressive kidney function decline, despite optimization of her blood pressure and diabetes, and 5 months later decompensated and ended on renal replacement therapy. A definitive, pharmacotherapeutic treatment for FGN is still unavailable, with transplant exhibiting high recurrence rates (35\%) in the limited population size of which it has been studied [7].

\section{Statement of Ethics}

The patient has agreed for clinical data to be submitted for a potential publication and signed the consent form. Written informed consent was obtained from the patient for publication of this case report.

\section{Disclosure Statement}

The authors declare that they have no competing interests.

\section{Author Contributions}

C.T. wrote the initial draft. B.P. and P.D. edited and revised the drafts. All authors agree with the content in the final draft.

\section{References}

1 Rosenstock JL, Markowitz GS, Valeri AM, Sacchi G, Appel GB, D’Agati VD. Fibrillary and immunotactoid glomerulonephritis: distinct entities with different clinical and pathologic features. Kidney Int. 2003 Apr;63(4):1450-61.

2 Javaugue V, Karras A, Glowacki F, McGregor B, Lacombe C, Goujon JM, et al. Long-term kidney disease outcomes in fibrillary glomerulonephritis: a case series of 27 patients. Am J Kidney Dis. 2013 Oct;62(4):67990 .

3 Iskandar SS, Falk RJ, Jennette JC. Clinical and pathologic features of fibrillary glomerulonephritis. Kidney Int. 1992 Dec;42(6):1401-7.

4 Alpers CE, Kowalewska J. Fibrillary glomerulonephritis and immunotactoid glomerulopathy. J Am Soc Nephrol. 2008 Jan;19(1):34-7.

5 Timmermans SA, Abdul-Hamid MA, Vanderlocht J, Damoiseaux JG, Reutelingsperger CP, van Paassen P, et al.; Limburg Renal Registry. Patients with hypertension-associated thrombotic microangiopathy may present with complement abnormalities. Kidney Int. 2017 Jun;91(6):1420-5.

6 Nasr SH, Valeri AM, Cornell LD, Fidler ME, Sethi S, Leung N, et al. Fibrillary glomerulonephritis: a report of 66 cases from a single institution. Clin J Am Soc Nephrol. 2011 Apr;6(4):775-84.

7 González-Cabrera F, Henríquez-Palop F, Ramírez-Puga A, Santana-Estupiñán R, Plaza-Toledano C, AntónPérez G, et al. The occurrence or fibrillary glomerulonephritis in patients with diabetes mellitus may not be coincidental: a report of four cases. Case Rep Med. 2013;2013:935172. 


\section{Case Reports in Nephrology and Dialysis}
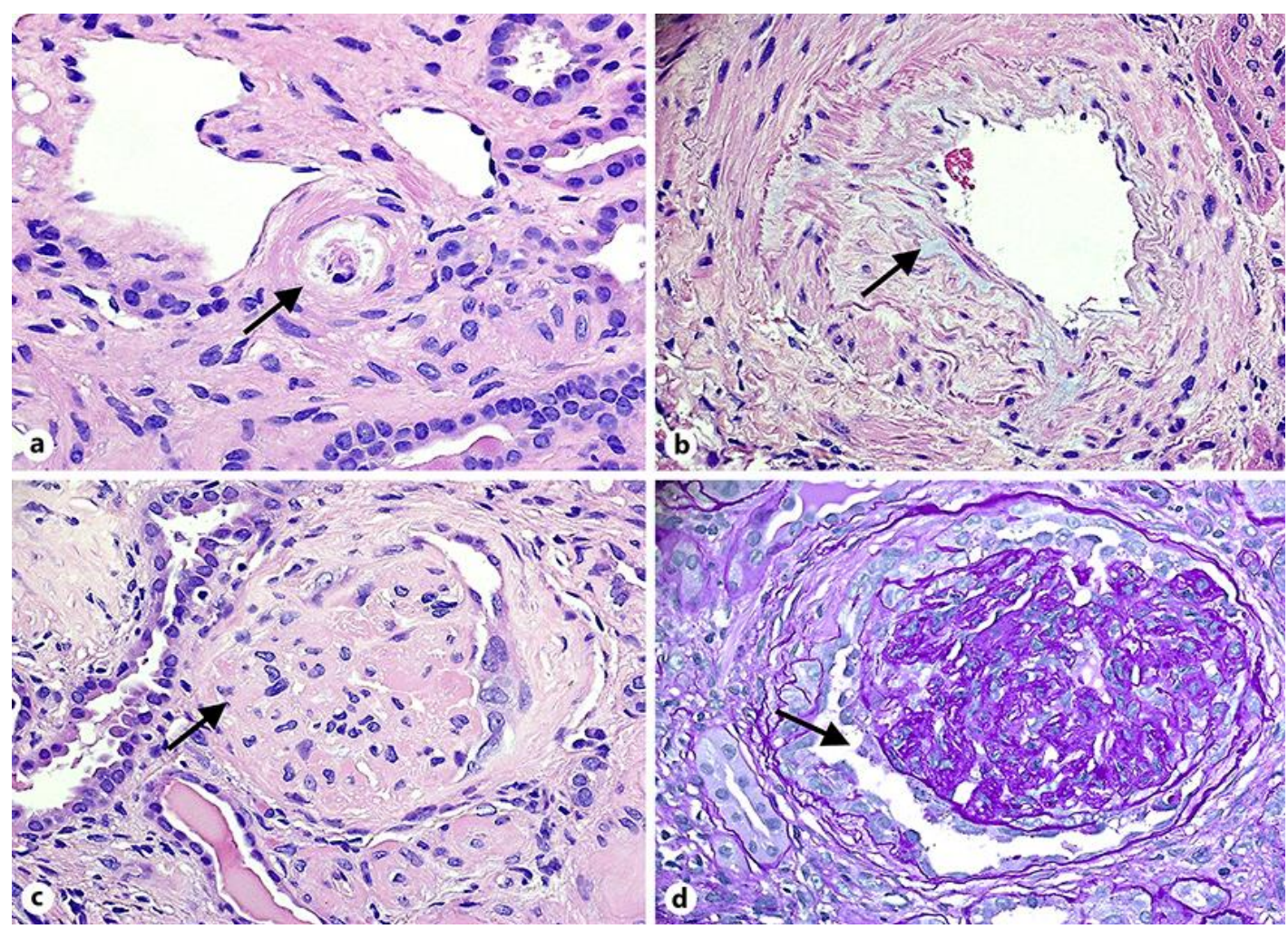

Fig. 1. Representative photomicrographs of the first biopsy demonstrate severe mucoid thickening of intimal layers of both arterioles and arteries ( $\mathbf{a}, \mathbf{b}$, marked by arrow), severely narrowing the lumen of these vessels. Normocellular glomeruli exhibiting expansion of PAS-positive matrix (c, marked by arrow) and thickening of glomerular basement membrane accompanied by collapsed capillary loops and prominence of epithelial cells (d, marked by arrow). 


\section{Case Reports in Nephrology and Dialysis}

Case Rep Nephrol Dial 2018;8:182-191

(C) 2018 The Author(s). Published by S. Karger AG, Basel DOI: $10.1159 / 000492529$ www.karger.com/cnd

Tsui et al.: FGN with Crescentic and Necrotizing Glomerulonephritis and Concurrent TMA
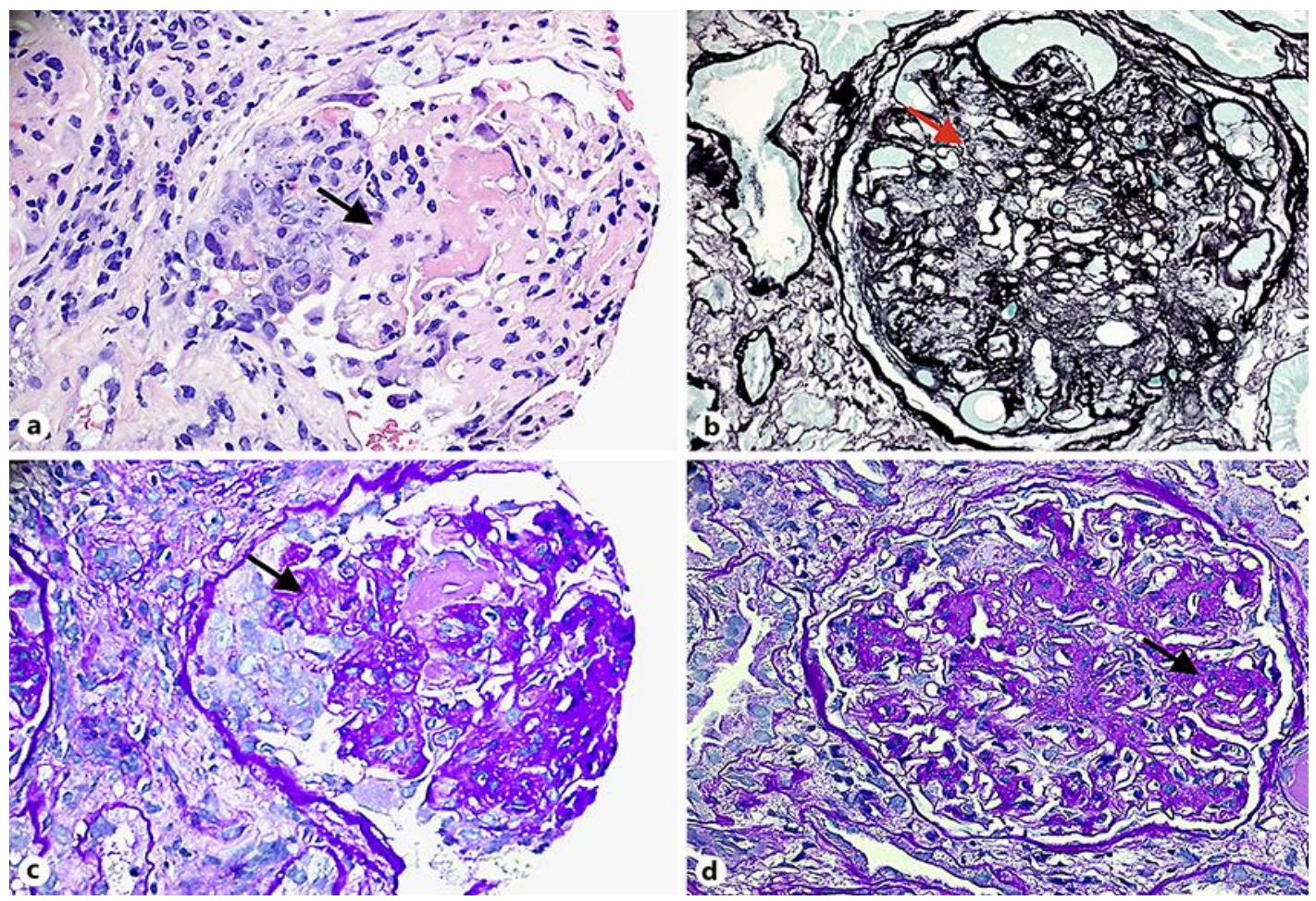

Fig. 2. Representative photomicrographs of the second biopsy demonstrate segmental necrotizing and crescentic lesion (a, marked by arrow) with accumulation of PAS-positive, partially silver-negative material in the mesangium (b, c, marked by arrow). Most glomeruli show nodular sclerotic lesions (d, marked by arrow) characteristic of diabetic nephropathy. 

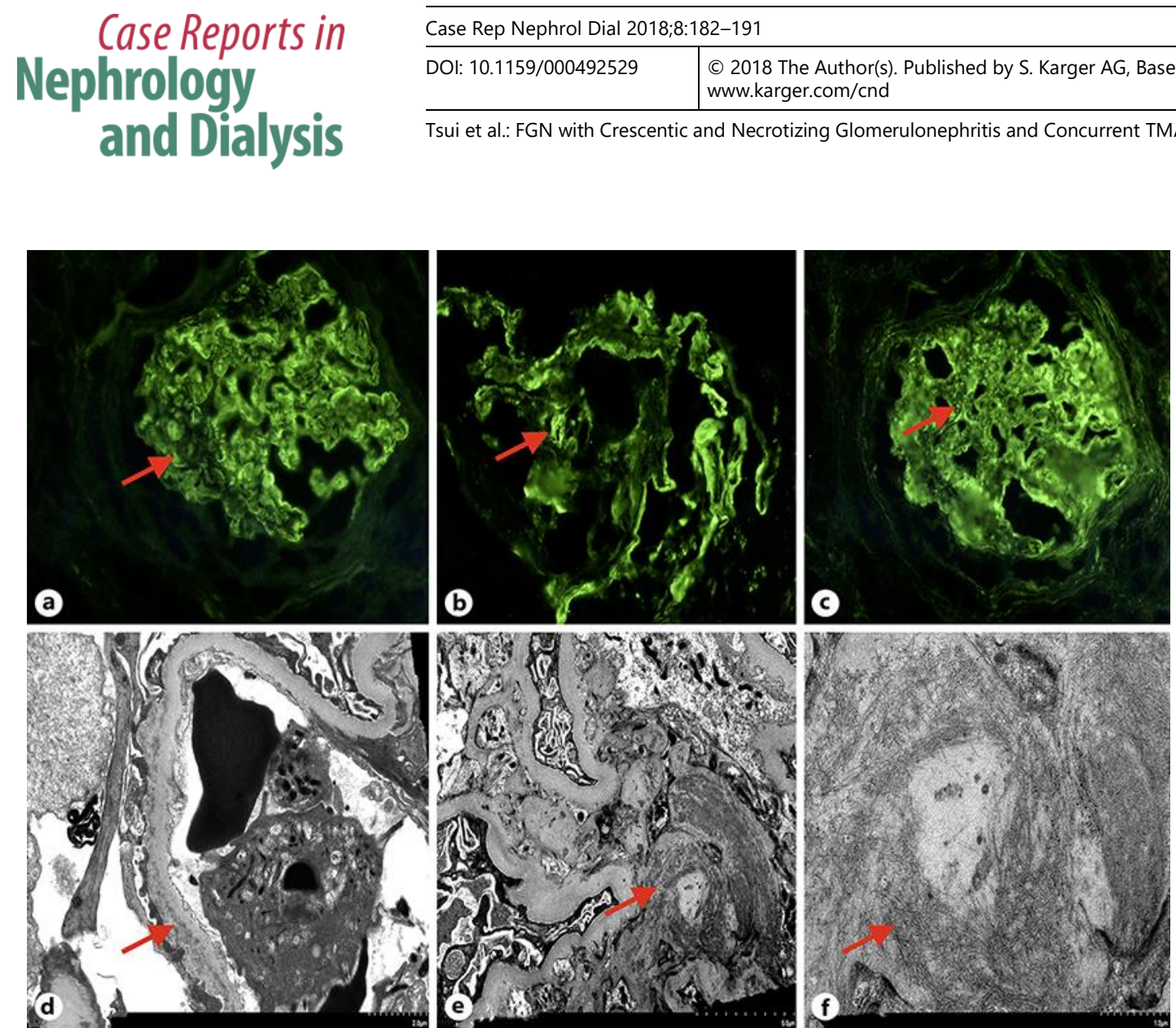

Fig. 3. Immunofluorescence microscopy shows deposition of IgG, C3, and C1q mainly on the capillary wall and also in the mesangium (a-c, marked by arrow). Images of electron microscopy demonstrate deposition of randomly arranged fibrils both in peripheral glomerular basement membranes (d, marked by arrow) and the mesangium (e, marked by arrow). Higher magnification of the randomly arranged non-branching fibrils deposited in the mesangial area (f, marked by arrow). 


\section{Case Reports in Nephrology and Dialysis}

Case Rep Nephrol Dial 2018;8:182-191

(C) 2018 The Author(s). Published by S. Karger AG, Basel DOI: $10.1159 / 000492529$ www.karger.com/cnd

Tsui et al.: FGN with Crescentic and Necrotizing Glomerulonephritis and Concurrent TMA
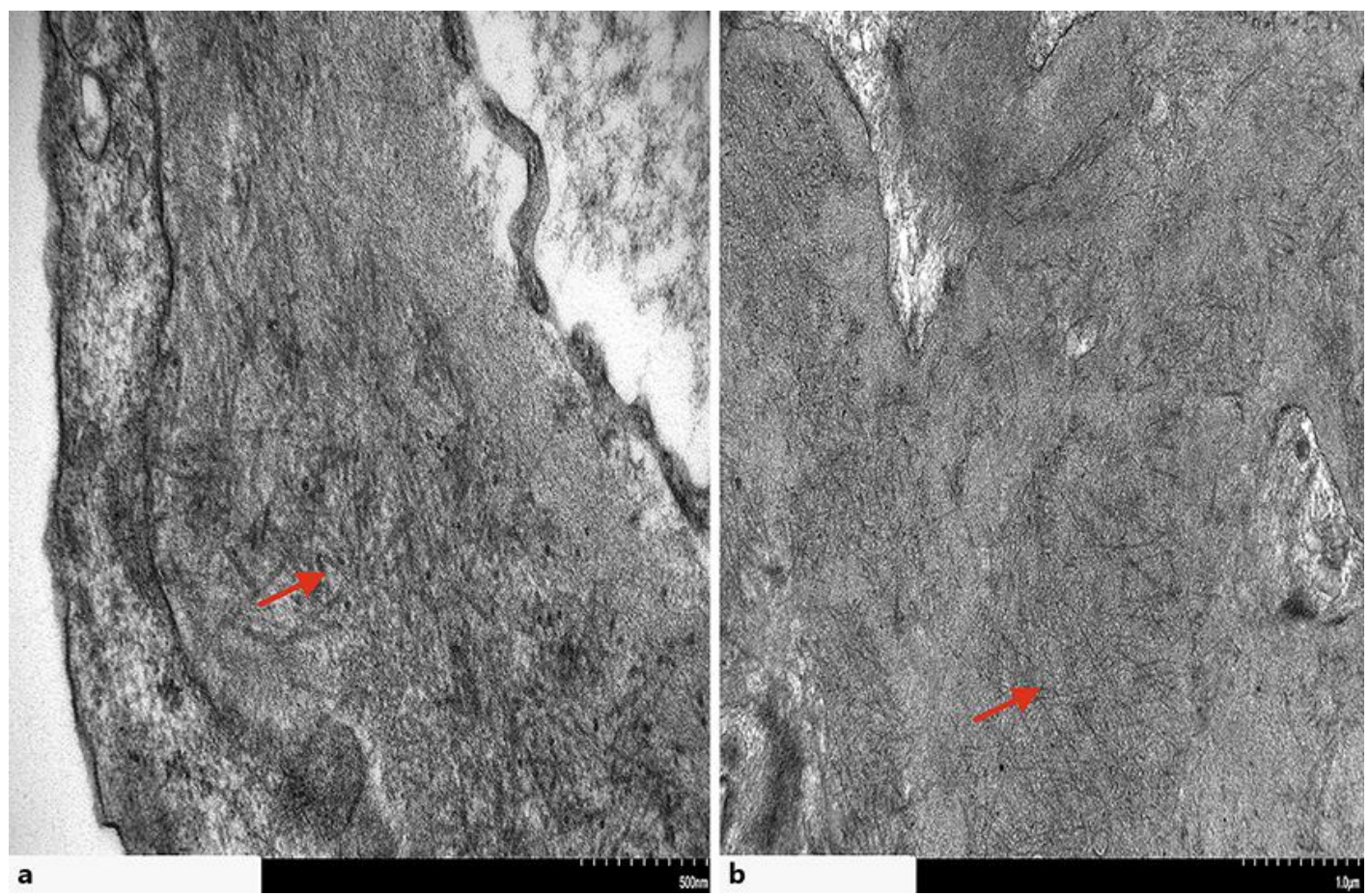

Fig. 4. Higher magnification $(\times 20,000)$ of the randomly arranged deposited fibrils in glomerular basement membrane (a, marked by arrow) and the mesangium (b, marked by arrow). The average thickness of fibrils is $12.4 \mathrm{~nm}$. 


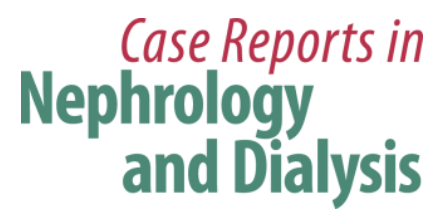

Case Rep Nephrol Dial 2018;8:182-191

Table 1. Baseline lab investigations

\begin{tabular}{lll}
\hline Test & Result & Reference range \\
\hline ESR & 35 & $0.74-1.85 \mathrm{~g} / \mathrm{L}$ \\
C3 & $1.49 \mathrm{~g} / \mathrm{L}$ & $0.16-0.44 \mathrm{~g} / \mathrm{L}$ \\
C4 & $0.40 \mathrm{~g} / \mathrm{L}$ & \\
Cryoglobulins & negative & \\
Anti-myeloperoxidase & negative & \\
Anti-proteinase 3 & negative & \\
Anti-GBM & negative & \\
Hepatitis B surface antigen & negative & \\
Hepatitis B surface antibody & $<3.1 \mathrm{IU} / \mathrm{L}$ & \\
Hepatitis C antibody & Negative & \\
Serum protein electrophoresis & No paraprotein band seen & $<150 \mathrm{mg} / \mathrm{day}$ \\
Urine protein electrophoresis & No Bence Jones protein & $3.3-19.4 \mathrm{mg} / \mathrm{L}$ \\
Urine protein & $6.6 \mathrm{~g} / \mathrm{day}$ & $5.7-26.3 \mathrm{mg} / \mathrm{L}$ \\
Serum $\kappa$ light chains & $107.0 \mathrm{mg} / \mathrm{L}$ & \\
Serum $\lambda$ light chains & $66.6 \mathrm{mg} / \mathrm{L}$ & \\
\hline
\end{tabular}

Table 2. Renal biopsy findings

\begin{tabular}{ll}
\hline Biopsy & Findings \\
\hline Light microscopy & There was segmental fibrinoid necrosis with overlying cellular crescent. \\
& $\begin{array}{l}\text { Mesangial matrix expansion forming vague nodularity. } \\
\text { Capillary lumens were narrowed by excessive matrix and enlarged endothelial } \\
\text { cells but no significant endocapillary proliferation was noted. }\end{array}$ \\
\hline Immunofluorescence & 6 glomeruli (2 globally and 2 segmentally sclerosed). \\
& Segmental smudgy staining of IgG, C3, and C1q in capillary wall and mesan- \\
& gium. \\
\hline Electron microscopy & Diffuse effacement of podocyte foot processes. \\
& $\begin{array}{l}\text { Diffuse thickening of basement membrane and mesangial matrix expansion. } \\
\text { Multiple irregular spaced subepithelial and intramembranous deposits of fi- } \\
\text { brillary aggregates. } \\
\text { The fibrils were haphazardly arranged and composed of non-branching fila- } \\
\text { ments (average diameter of 12.4 nm). }\end{array}$ \\
\hline
\end{tabular}

